\title{
Tunability of critical Casimir interactions by boundary conditions
}

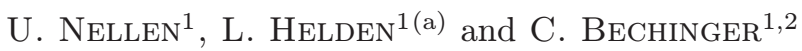 \\ 1. Physikalisches Institut, Universität Stuttgart - Pfaffenwaldring 57, 70550 Stuttgart, Germany, EU \\ ${ }^{2}$ Max-Planck-Institut für Metallforschung - Heisenbergstr. 3, 70563 Stuttgart, Germany, EU
}

\author{
PACS 68.35.Rh - Phase transitions and critical phenomena \\ PACS 82.70.Dd - Colloids \\ PACS 81.16.Dn - Self-assembly
}

\begin{abstract}
We experimentally demonstrate that critical Casimir forces in colloidal systems can be continuously tuned by the choice of boundary conditions. The interaction potential of a colloidal particle in a mixture of water and 2,6-lutidine has been measured above a substrate with a gradient in its preferential adsorption properties for the mixture's components. We find that the interaction potentials at constant temperature but different positions relative to the gradient continuously change from attraction to repulsion. This demonstrates that critical Casimir forces respond not only to minute temperature changes but also to small changes in the surface properties.
\end{abstract}

In 1978 Fisher and de Gennes pointed out that if two objects are immersed in a fluid close to its critical point, long-ranged forces due to confined critical fluctuations act between their surfaces [1]. Such critical Casimir forces arise due to the confinement of fluctuations in the order parameter of the fluid between the objects. In the case of e.g. a classical binary liquid mixture close to its demixing point, the order parameter corresponds to the concentration difference between the two components of the mixture. The strength and range of critical Casimir forces is set by the fluid's bulk correlation length $\xi$ which diverges upon approaching the critical temperature $T_{C}$. Therefore, close to $T_{C}$, the interaction strongly depends on the temperature as has been recently confirmed in several experiments [2-9].

In addition to their temperature dependence, critical Casimir forces are very sensitive to the boundary conditions (BC) which are determined by the adsorption preferences of the mixture's components at the confining surfaces: not only the magnitude, but even the sign of critical Casimir interactions can be altered by corresponding symmetric or asymmetric BC. So far, theoretical studies largely concentrated on $\mathrm{BC}$, where one species of molecules in the binary liquid mixture forms a saturated monolayer at the confining surfaces $[10,11]$. Depending on whether both surfaces strongly adsorb the same $(--)$ or different species $(-+)$, this results in attractive or repulsive

(a) E-mail: L.Helden@physik.uni-stuttgart.de forces which have been recently observed in several experiments $[3-8,12,13]$.

In this letter we report the first critical Casimir measurements for continuously tunable boundary conditions. This has been achieved by measuring the interaction energy of a single colloidal particle suspended in a critical water-2,6-lutidine mixture above a solid surface with a gradient in its adsorption preference for the two liquid components. Upon lateral displacement of the particle relative to the substrate we find a smooth transition from attractive to repulsive critical Casimir forces. The observed scaling functions are found to lie between that of the limiting cases of $(--)$ and $(-+)$ BC.

Surfaces with a spatial variation of adsorption preference for lutidine and water molecules were fabricated by immersing hydrophilic silica substrates into a mixture (1300:1) of hexane and octadecyltrichlorosilane (OTS). After about 30 minutes, a monolayer of OTS molecules binds to the surface and thus renders it hydrophobic [14]. Measurements of the contact angle confirm that this treatment alters the adsorption preference from that of water to lutidine. We obtained samples with a smooth lateral gradient regarding the OTS coverage by partially shielding the substrate with a thin metal blade and exposing it to an oxygen-nitrogen plasma, so that OTS molecules are fractionally removed. This gradient can be visualized by cooling the sample below the dew point. The corresponding breath figure (fig. 1a) shows small droplets with a large contact angle on the hydrophobic side where the sample was fully covered by the blade (right) and much larger 


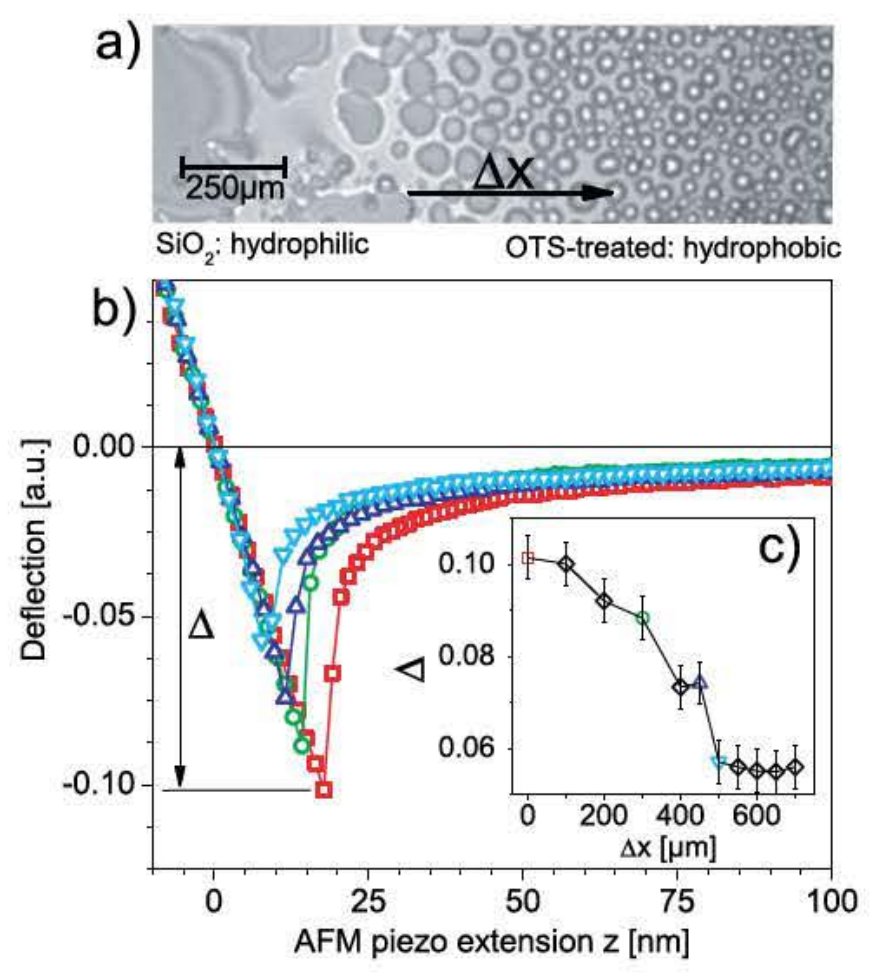

Fig. 1: (Color online) (a) Water droplets on a silica substrate with a gradient in its wetting properties below the dew point (breath figure). Small and large droplets indicate hydrophobic and hydrophilic regions. (b) AFM force-distance curves obtained with a hydrophilic cantilever at different positions $\Delta x$ on the surface. Curves were arranged such that the regions of constant compliance overlap at zero deflection signal. (c) Attraction strength $\Delta v s . \Delta x$ along the chemical gradient. Symbols correspond to those used in (b).

droplets with small contact angles (left) where the OTS molecules were removed and the sample is hydrophilic. The gradient in the surface properties was further characterized by force-distance curves obtained with an atomic force microscope (AFM) under ambient conditions and a freshly plasma-cleaned hydrophilic tip. Upon approaching the surface, at small distances the tip is suddenly attracted towards the surface due to van der Waals and capillary forces (fig. 1b). The strong contribution of capillary forces is supported by the fact that both strength $\Delta$ and range of the attraction decrease towards the hydrophobic side, i.e. with increasing $\Delta x$ (fig. 1c). Similar to the breath figures, the AFM measurements show that the above method yields substrates with smooth chemical gradients which laterally extend over a distance of several hundreds of microns.

We fabricated thin sample cells $(150 \mu \mathrm{m}$ height) with the described substrates as the bottom plate and inserted a diluted suspension of colloidal particles in a water2,6-lutidine (WL) mixture at critical composition, i.e. a lutidine mass fraction of $c_{L}^{c} \approx 0.286$. Such mixtures have a lower critical point at $T_{C} \approx 307 \mathrm{~K}$ [15]. As colloids we used negatively charged melamine spheres (MF) with radius
$R=1.35 \mu \mathrm{m}$ (see footnote ${ }^{1}$ ). Due to their high surface charge density the particles are strongly hydrophilic, i.e. they show a preference for water adsorption.

Interaction potentials between a single colloid and a substrate were measured with total internal reflection microscopy (TIRM). The entire sample cell was mounted onto a glass prism such that an incident p-polarized laser beam $(\lambda=473 \mathrm{~nm}, P \approx 2 \mathrm{~mW})$ is totally reflected at the substrate-fluid interface. Under these conditions an evanescent field is created which exponentially decays into the fluid. Our experiments were performed with a penetration depth of $153 \mathrm{~nm}$ by adjusting the angle of incidence accordingly. When the height $z$ of the colloid above the surface is in the region illuminated by the evanescent field, it will partially scatter the evanescent light. For the chosen conditions, evanescent light scattering on critical fluctuations in the mixture can be neglected compared to the light scattered by the colloidal particle. From the scattered intensity, which is monitored with a photomultiplier, the particle-substrate height distribution $P(z)$ can be inferred. Employing the Boltzmann factor, the heightresolved interaction potential for a colloid close to the substrate is derived. The lateral motion of the particle was reduced to about $\pm 1 \mu \mathrm{m}$ with a weakly focussed laser beam $(\lambda=532 \mathrm{~nm})$ acting as an optical tweezers from above. Since this value is orders of magnitude smaller than the lateral extension of the chemical gradient, the boundary conditions can be considered as homogeneous on the area probed during a single measurement. For further details regarding TIRM and the experimental setup we refer to the literature $[2,16,17]$.

Temperature control of the sample cell was achieved by a two-step procedure. We connected the sample with a copper frame to a heat bath operated at a constant temperature slightly below $T_{C}$. In addition, we used an electrical heater, which was connected to a temperature controller. In contrast to previous experiments, where the temperature of the binary liquid mixture was stabilized with respect to a platinum resistor placed outside the liquid, here the light scattering intensity from the critical fluctuations was used as input for the temperature controller [18]. For this purpose an additional laser beam $(\lambda=658 \mathrm{~nm})$ was coupled into the cell to propagate parallel to the substrate in the fluid. With this setup we achieved a temperature stability of about $\pm 2 \mathrm{mK}$. Since the scattering signal tends to diverge at the critical temperature, we can determine $T_{C}$ with a significantly improved accuracy of $\pm 5 \mathrm{mK}$.

The inset of fig. 2 (upper curve) shows a typical interaction potential between a single MF particle and a surface far below $T_{C}$ where critical Casimir forces are negligible. The shape of the potential can be fitted to

$$
\Phi(z)=A \exp (-\kappa z)+G^{*} z
$$

${ }^{1} \mathrm{MF}-\mathrm{COOH}-\mathrm{S} 1285, R=1.35 \pm 0.05 \mu \mathrm{m}$ microparticles $\mathrm{GmbH}$, Berlin, Germany. According to the manufacturer the surface potential in water is $70-100 \mathrm{mV}$. 


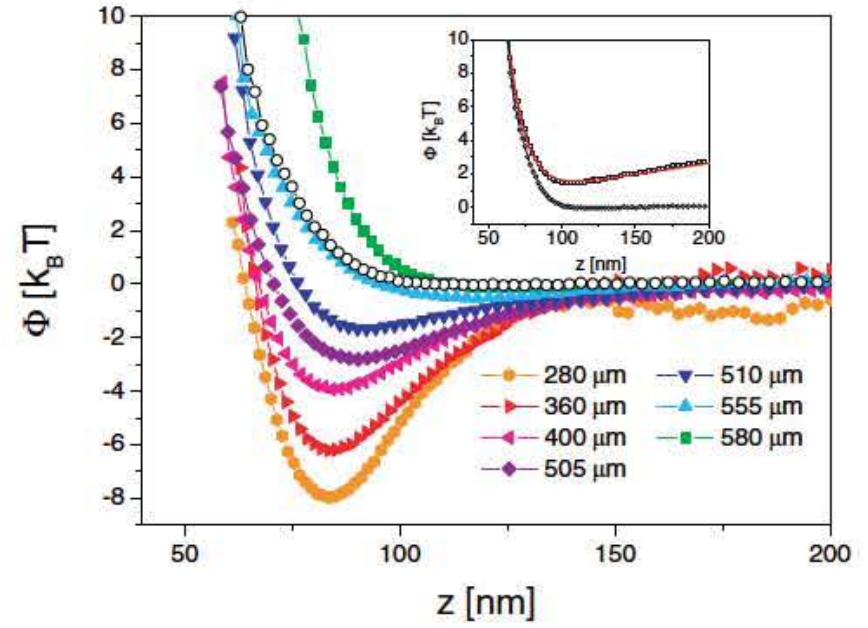

Fig. 2: (Color online) Interaction potentials between a MF particle and a silica substrate with a chemical gradient, obtained at different lateral positions $\Delta x$. Closed symbols were taken at $T_{C}-T=220 \mathrm{mK}$ corresponding to $\xi=19 \mathrm{~nm}$. The open symbols show a measurement for $T_{C}-T=1.50 \mathrm{~K}$ where critical Casimir forces are negligible. Inset: interaction potential as measured (squares) and after subtraction of the linear contributions (circles) which are due to optical and gravitational forces. The solid line is a fit to eq. (1) with $A=2770 k_{B} T, \kappa^{-1}=11.1 \mathrm{~nm}$ and $G^{*}=13.5 k_{B} T / \mu \mathrm{m}$.

with $A$ the amplitude of electrostatic interactions between the negatively charged particle and substrate, $\kappa$ the inverse Debye screening length of the mixture and $G^{*}$ the effective weight of the colloid due to gravity and light pressure from the optical tweezers. Since the linear contribution from gravitational and optical forces does not vary between individual measurements, in the following it has been subtracted from all data in this paper. Since the particlewall interaction potential (fig. 2 inset) is well fitted by eq. (1) and parameters in agreement with literature values $[19,20]$, possible contributions from van der Waals forces are negligible in the present experiment. A more detailed discussion can be found in $[21,22]$.

Interaction potentials at constant temperature $T_{C}-T=$ $220 \mathrm{mK}$ and different lateral positions $\Delta x=x-x_{0}$ relative to the substrate (with $x_{0}$ a reference position at the strongly hydrophilic site of the gradient) are shown in fig. 2. Since the colloidal particle is strongly hydrophilic, symmetric $\mathrm{BC}$ should apply at small values of $\Delta x$, i.e. on the strongly hydrophilic side. Under these conditions critical Casimir forces are attractive. In combination with the short-ranged electrostatic force, this leads to potential wells with depths of several times the thermal energy $k_{B} T$. With increasing $\Delta x$, i.e. upon approaching the hydrophobic side of the gradient, the $\mathrm{BC}$ become increasingly asymmetric and the critical Casimir forces become weaker. Accordingly, the potential wells become shallower and are shifted towards larger distances. Close to the hydrophobic region of the gradient lutidine is

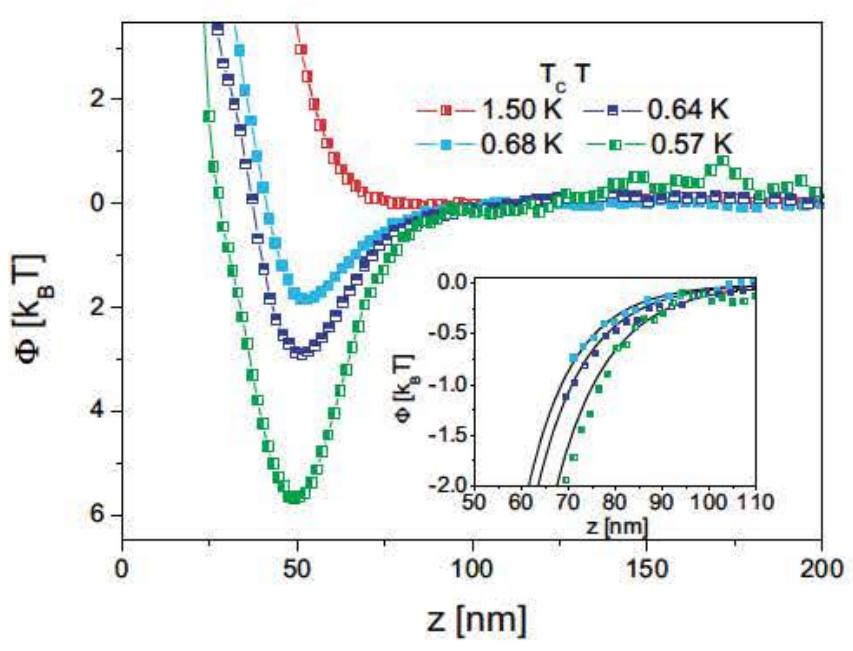

Fig. 3: (Color online) Temperature dependence of critical Casimir forces at fixed position $\Delta x=0$. The inset shows the distance range where electrostatic interactions are negligible. Fits to theoretical predictions for (--) BC are shown as solid lines.

preferred by the substrate and the critical Casimir forces should be repulsive. Indeed for $\Delta x=580 \mu \mathrm{m}$, such a repulsion is observed in our data, as can be seen by direct comparison with the particle-wall interaction potential far below $T_{C}$ (open symbols) where critical Casimir forces are negligible.

For $(--)$ and $(-+)$ BC the critical Casimir potential of a colloidal sphere with radius $R$ at height $z$ above a homogeneous surface is given by $[2,10]$

$$
\Phi_{C a s}(z, T)=\frac{R}{z} \vartheta\left(\frac{z}{\xi}\right)
$$

with the correlation length

$$
\xi=\xi_{0}\left(\frac{T_{C}-T}{T_{C}}\right)^{-\nu},
$$

$\xi_{0}$ reflecting the typical length scale set by the intermolecular pair potential in the mixture, $\nu=0.63$ the critical exponent of the 3D Ising universality class and $\vartheta$ the corresponding scaling functions which have been inferred from Monte Carlo simulations $[23]^{2}$. To confirm, that the potentials indeed result from critical Casimir forces, we first investigated the temperature dependence of the potential at $(\Delta x=0)$ where $(--)$ BC apply (fig. 3). In the inset we show the experimental data for the region where electrostatic and van der Waals interactions are negligible. As solid lines we plotted the fits according to eq. (2) which show good agreement. It should be emphasized that

\footnotetext{
${ }^{2}$ The Derjaguin approximation was used to adapt simulation results for wall-wall geometry to the sphere-wall geometry of the experiment. This is justified since $R$ is much larger than its distance $z$ and the maximum correlation length $\xi_{\max }=40 \mathrm{~nm}$.
} 


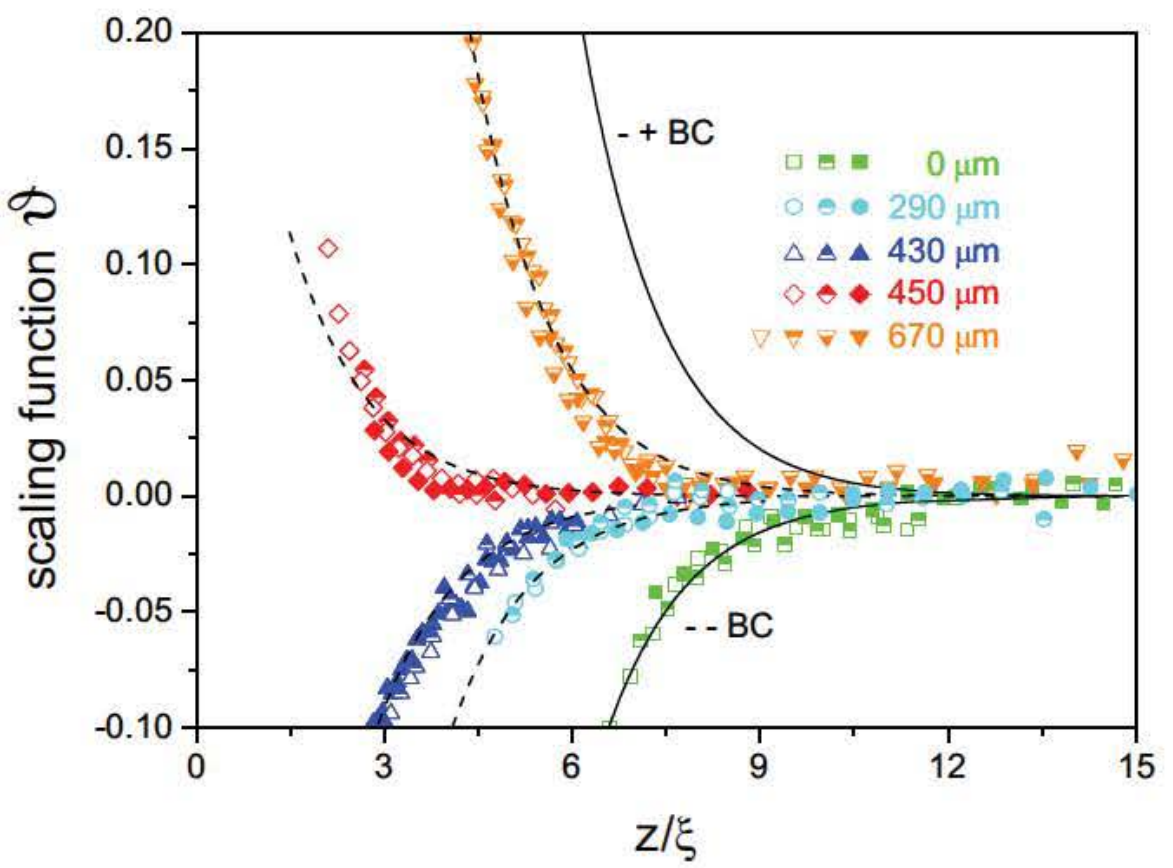

Fig. 4: (Color online) Measured scaling functions for different lateral positions $\Delta x$ on the substrate. Symbols of the same kind but with different fillings correspond to measurements at different temperatures and collapse to a single curve each. The square symbols were determined from the corresponding curves in fig. 3 . Theoretical calculations for the limiting cases of ( --$)$ and $(-+) \mathrm{BC}$ are shown as solid black lines $[2,23]$. Dashed lines represent the same curves shifted along the $\frac{z}{\xi}$-axis to obtain the best agreement with data for weaker adsorption preference.

only $\xi_{0}$ has been used as an adjustable parameter. The best agreement with our experimental data was found for $\xi_{0} \approx 0.2 \mathrm{~nm}$, which is in good agreement with other measurements in critical water-lutidine mixtures [21,24].

According to eq. (2) the information about the $\mathrm{BC}$ is entirely encoded in the scaling function $\vartheta$. Therefore, we determined $\vartheta$ from the measured critical Casimir interaction potentials for different substrate positions $\Delta x$ (fig. 4). Note that symbols with identical shape but different fillings correspond to scaling functions obtained at the same position $\Delta x$ but for different temperatures. Data taken at different temperatures collapse onto a single curve in this representation. With increasing $\Delta x$ the scaling functions change systematically from negative to positive values. This is consistent with the sign change of critical Casimir interactions observed along the chemical gradient as shown in fig. 2. For comparison we added as solid lines the theoretical predictions for the scaling functions for $(--)$ and $(-+)$ BC. As can be seen, the measured values for $\vartheta$ lie in between these limiting cases. On the hydrophilic side of the substrate we obviously reached $(--)$ BC while we did not reach $(-+)$ $\mathrm{BC}$ on the hydrophobic side. This indicates that the lutidine adsorption on the OTS treated substrate is not saturated.

Scaling behavior is observed for all positions $\Delta x$ which is not a priori clear because eq. (2) is strictly valid only for
$(--)$ and $(-+)$ BC [25]. This indicates that additional scaling variables which may arise in the presence of undersaturated adsorption layers are not relevant for data collapse at the $\frac{z}{\xi}$ range sampled in our experiments [26]. Mean-field theory calculations predict that the scaling functions for $\mathrm{BC}$ close to the strong adsorption limit can be obtained by a shift along the $\frac{z}{\xi}$-axis $[27,28]$. This is in remarkable agreement with the dashed lines in fig. 4 which just correspond to shifted $\vartheta$ functions for $(--)$ and $(-+)$ $\mathrm{BC}$ obtained by a least mean square fit to the data.

At present, it remains unclear how experimentally accessible parameters for the quantitative characterization of boundary conditions can be related to, e.g., the surface field $h_{1}$ which is often used to theoretically describe continuously varying BC [29-31]. Ellipsometry studies on critical adsorption of binary mixtures under weak surface field conditions suggest that $h_{1}$ is proportional to the surface energy difference of the two liquid components [26], while other approaches tried to connect the surface field with the difference in the chemical potential [30]. We hope that our work will stimulate further theoretical investigations in this direction.

In summary, we have shown that critical Casimir forces can be continuously varied by appropriate BC of the confining surfaces. Experimentally, this was achieved by lateral variation of the surface coverage of a single layer of OTS molecules on the substrate which leads both to a change of the magnitude and the sign of critical Casimir 
forces between a colloidal particle and the surface. In addition to the exquisite temperature dependence, this remarkable sensitivity on the surface properties of the interacting objects distinguishes critical Casimir forces as a versatile interaction type which adds novel perspectives to the use of colloidal suspensions as model systems but also opens new possibilities for the fabrication of colloidal crystals which hold significant interest for technical applications.

We thank S. Dietrich, A. MaciołeK, T. Mohry, and A. GAmBAssi for stimulating discussions, T. Geldhauser for assistance with AFM measurements and the Deutsche Forschungsgemeinschaft for financial support.

\section{REFERENCES}

[1] Fisher M. E. and DE Gennes P. G., C. R. Hebd. Seances Acad. Sci. Ser. B, 287 (1978) 207.

[2] Hertlein C., Helden L., Gambassi A., Dietrich S. and Bechinger C., Nature, 451 (2008) 172.

[3] Ganshin A., Scheidemantel S., Garcia R. and Chan M. H. W., Phys. Rev. Lett., 97 (2006) 075301.

[4] Fukuto M., Yano Y. F. and Pershan P. S., Phys. Rev. Lett., 94 (2005) 135702.

[5] Garcia R. and Chan M. H. W., Phys. Rev. Lett., 88 (2002) 086001.

[6] Garcia R. and Chan M. H. W., Phys. Rev. Lett., 83 (1999) 1187.

[7] Mukhopadhyay A. and Law B. M., Phys. Rev. Lett., 83 (1999) 772.

[8] Rafaï S., Bonn D. and Meunier J., Physica A, 386 (2007) 31.

[9] Gambassi A., Hertlein C., Helden L., Dietrich S. and Bechinger C., Europhys. News, 48 (2009) 18.

[10] Hanke A., Schlesener F., Eisenriegler E. and Dietrich S., Phys. Rev. Lett., 81 (1998) 1885.
[11] Krech M., Phys. Rev. E, 56 (1997) 1642.

[12] Soyka F., Zvyagolskaya O., Hertlein C., Helden L. and Bechinger C., Phys. Rev. Lett., 101 (2008) 208301.

[13] Tröndle M., Kondrat S., Gambassi A., Harnau L. and Dietrich S., preprint arXiv:0903.2113v1 (2009).

[14] Ulman A., Chem. Rev., 96 (1996) 1533.

[15] Beysens D. and Esteve D., Phys. Rev. Lett., 54 (1985) 2123.

[16] Walz J. Y., Curr. Opin. Colloid Interface Sci., 2 (1997) 600.

[17] Prieve D. C. and Frej N., Langmuir, 6 (1990) 396.

[18] Schrödle S., Buchner R. and Kunz W., Fluid Phase Equilib., 216 (2004) 175.

[19] Gallagher P. D. and Maher J. V., Phys. Rev. A, 46 (1992) 2012.

[20] von Grünberg H. H., Helden L., Leiderer P. and Bechinger C., J. Chem. Phys., 114 (2001) 10094.

[21] Gambassi A., MaciołeK A., Hertlein C., Nellen U., Helden L., Bechinger C. and Dietrich S., preprint arXiv:0908.1795v1 (2009).

[22] Dantchev D., Schlesener F. and Dietrich S., Phys. Rev. E, 76 (2007) 011121.

[23] Vasilyev O., Gambassi A., Maciolek A. and DiETRICH S., EPL, 80 (2007) 60009.

[24] Gülari E., Collings A. F., Schmidt R. L. and Pings C. J., J. Chem. Phys., 56 (1972) 6169.

[25] Schmidt F. M. and Dienl H. W., Phys. Rev. Lett., 101 (2008) 100601.

[26] Chо J. H. J. and Law B. M., Phys. Rev. E, 65 (2002) 011601.

[27] Binder K., in Phase Transitions and Critical Phenomena, edited by Domb C. and LeBowitz J. L., Vol. 8 (Academic, London) 1983, p. 1.

[28] Mohry T. F., MacioŁeK A. and Dietrich S., in preparation.

[29] Maciolek A., Evans R. and Wilding N. B., J. Chem. Phys., 119 (2003) 8663.

[30] Desai N. S., Peach S. and Franck C., Phys. Rev. E, 52 (1995) 4129.

[31] Durian D. J. and Franck C., Phys. Rev. Lett., 59 (1987) 555. 